\title{
Survey on Web Service Discovery and Availability in Mobile Environments and Future Trends in Mobile Web Services
}

\author{
Roshan Fernandes \\ NMAM Institute of Technology, \\ Nitte, India
}

\author{
Rio D'Souza, PhD \\ St Joseph Engineering College, \\ Vamanjoor, India
}

\begin{abstract}
The advancement in technology related to mobile devices has become a very important platform to share mobile information among millions of users across the globe. Also the mobile devices are easily available and accessible among the people. Recently, the mobile devices have been powered by faster and multicore processors, more available memory and sophisticated user display. People prefer smart phones rather than carrying laptops or desktops because of their small size, light weight and anywhere anytime information provider. Many users need to access the services on these mobile phones connecting through the web. Just like centralized service providers there is a great need of providing service through mobile phones. In most scenarios, personal smart phones also provide web services. These services are referred as mobile web services since the devices are in mobility. In this paper, a review on the availability and discovery of web services in resource constrained mobile specific environments and the future trends have been discussed.
\end{abstract}

\section{General Terms}

Mobile Web Services, Semantic and Syntactic Web Services, Mobility prediction

\section{Keywords}

SOAP, UDDI, WSDL, XMPP, OWL-S, SAWSDL, WSMO, REST

\section{INTRODUCTION}

Mobile devices may be used for accessing or discovering web services but little work has been done to enable hosting them on the mobile devices. Hosting of web services on resource constrained mobile devices has got several challenges. The challenges include the battery power, the network constraints, limited computational power of mobile device, mobile service registry management, dependency on web service architectures which involves SOAP (Simple Object Access Protocol), UDDI (Universal Description, Discovery and Integration), WSDL (Web Service Description Language).

When there is increasing number of personalized and dynamic web services hosted over the mobile devices, identifying and discovering such service becomes a major issue. Mobile devices which provide services may not be available every time because of the battery issues or due to network outage. In such a scenario, centralized management of web services through a service registry namely, UDDI, is not feasible. There is a need to design a new light weight model to handle the services provided by modern smart mobile devices.

Most of the times to find a single service that satisfies a very specific request may not be possible. So the request has to be broken down into sub tasks and each sub task should find the service separately. The resulting response must be combined together to fulfil the initial request. The service discovery can be done through syntactic or semantic ways. The former technique works only on exact keyword matching there by missing many web services which may not be listed with the keyword. Semantic web service discovery come across this drawback. Unfortunately, the semantic match process is a high resource consuming activity and is best suited for deploying on high performance servers. There is a need for the highly optimized semantic match making process for mobile devices, where there is a limited hardware resource support available.

\section{EFFECTIVE WEB SERVICE DISCOVERY AND AVAILABILITY IN MOBILE ENVIRONMENTS}

In recent times, the Mobile technology is used by millions of people in the world. In this context the user may access the Web service at any time in any place. The researchers in the previous year have been focusing on optimizing the approaches for web service discovery that can fit into mobile domains. These include the mobility based services with respect to a specific place [1], with what context the mobile services to be discovered [2], knowing the capabilities of the device [3], discovering the services based on the capacity of the device [4], combining the different services in a mobility specification [5].

The Web Service Description standards ensure that the web services to interact seamlessly among different applications through standard protocols. WSDL 2.0 [6] is the standard recent specification for the non-semantic web services. It specifies the interface which combines the operations and services. The drawback is that it is based on exact operation names and messages.

There exist few semantic web service description languages. They include: Web Ontology Languages (OWL-S) [7], Semantic Web Services Ontology (SWSO) [8], Web Services Semantics (WSDL-S) [9], Web Service Modelling Ontology (WSMO) [10]. The drawback of the above mentioned semantic description languages is that, each of them has its own representation and there is no standard notations exist for the semantic descriptions.

Service discovery [20] is a process of getting a specific service response for a specific service request. There are three existing techniques for service discovery. The first is UDDI Business Registry (UBR). The UBR relies on the central storage repositories. These repositories are used by the providers to publish their specific business web services. The end users or customers use it to discover specific business web services that they need for their purpose. The drawback of UBR includes the centralized architecture, failure of centralized server, searches that specifically depend on service keywords, outdated service records, limited scalability. Another major setback is that the customer needs to know the 
address of UBR in order to identify its location and send request to it.

The second is the Specialized Search Engines. They collect the web service description files from the public UBRs and from different web contents. Once these description files are received, the engines extract the meaning of web services from the description files and then perform semantic match between the requests and web service descriptions. Examples include Woogle and WSCE. The major challenge is that the retrieved services should be valid.

The third technique is Generic Search Engines. In this technique, the customers can use the search engines to find the required web services by writing a customized request in the form of a query. The main drawback of this technique is that they cannot understand the functions of the web services specified in the service description files. They depend only on the specific service keywords to identify services. The major advantage is that it is robust, scalable and no additional infrastructure is needed.

Khalid Elgazzar, Hossam Hassanein, Patrick Martin developed a framework for discovery of web services for the mobile environments [11]. It is a 3 - layered architecture where the layer 1 represents the components which reside on the customer side, layer 2 represents some components that can be implemented either on the service provider side or on the consumer side depending on the availability of resource and battery power. Layer 3 represents the components that execute on service provider side. The customer layer prepares a request for the required service which fulfils the objective. At the layer 2, the request analyzer uses the Request Submission Protocol to search atomic services which completely respond to user's request. If no relevant service found, it breaks down the request into sub tasks and each sub task is handled separately. It also ranks the list of relevant web services based on user specifications, ratings, preferences and execution environments. The framework also stores locally the retrieved web services for the further choosing of next best candidate service. The layer 3 matches the request with the web services. The authors use WSDL file, OWL-S based and WSMO based approaches. The drawback of this framework is that it is heavy and inefficient for resource constrained mobile devices. Also these approaches are not generic.

The authors of paper [12] proposed a lightweight client/server based architecture for providing and consuming mobile web services. In this work, a RESTful (Representational State Transfer) web service is implemented using HTTP. The authors claim that the direct implementation of SOAP based web services on mobile devices may result in the unacceptable level of overhead because of heavy SOAP request-response messages. These are too large and heavy to be installed into mobile devices which have got limited resources. On the other hand RESTful web services provide greater performance, efficient bandwidth and are robust. The paper implements a Dictionary web service that supports JSON (Java Script Object Notation) data representation. This implementation does not concentrate on real time service discovery and selection. It considers few specific dictionary words as the service request and the meaning as the service response. The advantage is that it is lightweight since it uses JSON.

Khalid Elgazzar, Hossam Hassanein, Patrick Martin [13] [14] describes the Personalized Mobile Web Service Discovery. In this work, the web service is deployed on the mobile device. In the device a light weight web server exists to provide the functionalities of HTTP - based service communications. In this architecture, the mobile device user is the service provider and the service consumer is a direct or indirect contact of the provider. The mobile service provider advertises web services to the members of his/her contact list. The service communication (request and response) is performed through a one to one communication between the provider and a consumer. The limitation of this approach is that the communication (request and response) is limited only to contact list addresses.

Rohit Verma, Abhishek Srivastava [15] proposed directory based architecture for the mobile web service request and response phases. In this paper, the authors focus on deploying service registry over mobile devices. The XMPP (eXtensible Messaging and Presence Protocol) is used for this purpose. The XMPP protocol is widely used for messaging and chatting applications using contact management and contact search. XMPP has been shown to be effective in managing the service directory, sending service updates and service availability information. Again this framework is limited to small users and also the authors not concentrating on how the available sensor services can be best utilized in a mobile environment.

Authors of paper [16] predict that in future the modern smart mobile devices will become the service providers. This is because of the presence of various sensors in mobile devices and each individual smart mobile device may contribute towards the providing of mobile web services. So the web service compositions must re think towards this issue. Unfortunately none of the above discussed papers present a solution to this issue.

\section{SEMANTIC WEB SERVICE DISCOVERY TOOLS}

There are three important and popular semantic web service discovery tools available. They are namely OWL-S (Web Ontology Language for Services), WSMO (Web Service Modelling Ontology) and SAWSDL (Semantic Annotations for WSDL).

The first one is OWL-S (Web Ontology Language for Services) [17] defines web service capabilities in a meaningful way in a web service open environment. The OWL-S classes are associated with three main aspects which include service profile, service model and service grounding. Meta data related to a specific service is specified by the service profile. This includes name of the service, inputs and preconditions and possible output. This meta information is used for the purpose of service discovery. The service model (also called process model) gives the details of various processes that are executing within a service. These processes can be either atomic or simple or composite. The atomic service is the one which can be executed in a single interaction. The simple services describe the service abstractions. These simple services can be implemented by an atomic process or service. The simple services may be extended to a composite service. The composite process consists of atomic and other composite processes. Grounding contains information on how to access a service, the message formats, transport protocol, addressing and serialization.

The second tool is WSMO (Web Service Modelling Ontology) [18] which provides a framework for describing web services in a semantic or meaningful way. It also allows the web service meta data model using Meta Object Facility (MOF). This may include dictionary of terms used to specify 
the web service description. The meta data model consists mainly of four elements. They are web services, goals, ontologies and mediators. The web services component specifies the aspects which are related to web service interaction. It also includes the functional properties and nonfunctional properties of the web service. The goals specify the target to be achieved by a client while using the described web service. The ontologies specify the terminology for the meta-model elements. The mediators provide support for connecting or linking various heterogeneous web service components and there by performing the transformation and reduction between the web services.

Another tool is the SAWSDL (Semantic Annotations for WSDL) [19] which extend the existing WSDL XML schema specifications. The semantic descriptions are added to the input and output messages, operations and interfaces. This semantically annotated WSDL or XML schema document can be published in the online registry and this service subsequently discovered, invoked or composed with other web services.

In summary, OWL-S and SAWSDL provides conceptual or high level semantic web service description support where as WSMO is a fully semantic web service framework having limited flexibility. The main benefit of SAWSDL is that it reuses the existing XML specification tools and technologies. In the current versions of all three tools mentioned above, they successfully provide the required response for effective semantic web service discovery and description from few specific viewpoints. But they fail from most other general viewpoints.

\section{DISCUSSION AND FUTURE TRENDS}

The implementation of web services on the moving resource constrained mobile devices is a challenging issue. Several works have been done to solve this issue but none of them give a complete solution. Several works have been done on the semantic web service composition. But there is always a challenge to do it on resource constrained mobile devices.

In the case of mobile web service discovery the user experience and satisfaction, specific user requirements, features of resource constrained mobile devices and capabilities are important factors that must be taken care of. Most of the research proposals fail to consider these issues.

The syntactic keyword based service discovery is not effective since some relevant services may not be responsive. There is a need for the efficient and light-weight semantic-based discovery in the resource constrained mobile devices.

Some times because of the mobile device service providers' mobility, the services offered by mobile devices may become invalid or not accessible. The main challenge is to ensure that the required services to be found are active and available always.

\section{CONCLUSION}

In this paper the existing work done on the effective web service discovery and availability in mobile environments has been discussed. The review on the existing semantic web service tools also discussed. Since the mobile devices are used for the personal use along with as a service provider, the security issues also should be taken care of. The solution to the mobility prediction issue will help the service providers and consumers to exchange service messages without interruption. Some of the identified issues have been discussed in section 4. There are still major issues to be identified in the area of mobile web services especially Quality of Service and the non-functional issues. There is need to explore these issues in the area of mobile web services.

\section{REFERENCES}

[1] E. Kaasinen, "User needs for location-aware mobile services", Personal and Ubiquitous Computing, Vol 7, pp. $70-79,2003$

[2] N. Blefari-Melazzi, E. Casaliccho and S. Salsano, "Context aware service dicovery in mobile heterogeneous environments" in mobile and wireless communications summit, 2007, $16^{\text {th }}$ IST, pp 1- 5, July 2007

[3] E. Al-Masri and Q. H. Mahmood, " Mobileureka: an approach for enhancing the discovery of mobile web services", Personal Ubiquitous Computing, Vol 14, pp 609-620, October 2010

[4] Z. Maamar, S. Tata, D. Belaid, and K. Boukadi, "Towards an approach to defining capacity-driven web service", International Conference on Advanced Information Networking and Applications (AINA'09), pp 403-410, 2009

[5] A. Bhuvaneswari and G. Karpagam, "Reengineering semantic web service composition in a mobile environment", International Test Conference, pp 227230,2010

[6] R. Chinnici, J-J Moreau, A. Ryman, and S. Weerawarana, "Web Service description language (WSDL) version 2.0 part 1: core language", June 26, 2007. http://www.w3.org/TR/wsdl20

[7] M. Burstein et. al. "OWL-s: Semantic markup for web servies", http://www.w3.org/Submission/OWL-S

[8] S. Battle et.al "Semantic web services ontology SWSO", W3C Member submission, 2005. http://www.daml.org/services/swsf/1.0/swso

[9] R. Akkiraju et. al, "Web services semantics - wsdl-s" W3C member submission, November 2005, http://www.w3.org/Submission/WSDL-S/

[10] D. Roman et. al., "Web Service modelling ontology", Applied Ontology, vol 1 pp 77-106, November 2005

[11] Khalid Elgazzar, Hossam Hassanein, Patrick Martin "Effective web service discovery in mobile environments", $7^{\text {th }}$ IEEE International Workshop on Performance and Management of Wireless and Mobile Networks, 2012

[12] KamalEldin Mohamed, Duminda W, "A lightweight framework for Web services implementations on Mobile Devices", 2012 IEEE First International Conference on Mobile Services, 2012

[13] Khalid Elgazzar, Hossam Hassanein, Patrick Martin "Towards personal mobile web services", 2013 IEEE Wireless Communications and Networking Conference (WCNC): Services and Applications, 2013

[14] Khalid Elgazzar, Hossam Hassanein, Patrick Martin "Personalized mobile web service discovery", 2013 IEEE Ninth World Congress on Services", 2013 
[15] Rohit Verma, Abhishek Srivastava, "A novel web service directory framework for mobile environments", 2014 IEEE International Conference on Web Services, 2014

[16] Yang Syu, Shang-Pin Ma, Jong-Yih Kuo, Yong-Yi FanJiang, "A survey on automated service composition methods and related techniques", 2012 IEEE Ninth International Conference on Services Computing, 2012

[17] A. Ankolekar, et. al., "DAML-S: Web Service Description for the Semantic Web", First International Semantic Web Conference (ISWC) Proceedings, 2002, pp. $348-363$
[18] D. Roman, et. al., "Web Services modelling ontology", Journal of Applied Ontology, vol. 39, no. 1, pp. 77-106, 2005

[19] J. Kopecky, et. al., "SAWSDL: Semantic Annotations for WSDL and XML Schema”, IEEE, pp. 60-67, Nov. 2007

[20] S. Hagemann, C. Letz, and G. Vossen, "Web service discovery - reality check 2.0," in Proceedings of the Third International Conference on Next Generation Web Services Practices, NWESP '07, pp. 113-118, 2007 